\title{
MutEnricher: a flexible toolset for somatic mutation enrichment analysis of tumor whole genomes
}

\author{
Anthony R. Soltis ${ }^{1,2^{*}}$, Clifton L. Dalgard ${ }^{1,3}$, Harvey B. Pollard ${ }^{1,3}$ and Matthew D. Wilkerson ${ }^{1,2,3^{*}}$ (D)
}

\author{
* Correspondence: anthony.soltis. \\ ctr@usuhs.edu; matthew.wilkerson. \\ ctr@usuhs.edu \\ ${ }^{1}$ The American Genome Center, \\ Collaborative Health Initiative \\ Research Program, Uniformed \\ Services University of the Health \\ Sciences, Bethesda, MD, USA \\ Full list of author information is \\ available at the end of the article
}

\begin{abstract}
Background: Analysis of somatic mutations from tumor whole exomes has fueled discovery of novel cancer driver genes. However, 98\% of the genome is noncoding and includes regulatory elements whose normal cellular functions can be disrupted by mutation. Whole genome sequencing (WGS), on the other hand, allows for identification of non-coding somatic variation and expanded estimation of background mutation rates, yet fewer computational tools exist for specific interrogation of this space.

Results: We present MutEnricher, a flexible toolset for investigating somatic mutation enrichment in both coding and non-coding genomic regions from WGS data. MutEnricher contains two distinct modules for these purposes that provide customizable options for calculating sample- and feature-specific background mutation rates. Additionally, both MutEnricher modules calculate feature-level and local, or "hotspot," somatic mutation enrichment statistics.

Conclusions: MutEnricher is a flexible software package for investigating somatic mutation enrichment that is implemented in Python, is freely available, can be efficiently parallelized, and is highly configurable to researcher's specific needs. MutEnricher is available online at https://github.com/asoltis/MutEnricher.
\end{abstract}

\section{Background}

Analysis of somatic mutations throughout the protein-coding genome, particularly via tumor whole exome sequencing (WES), has fueled the discovery of many cancer driver genes [1]. However, the vast majority of the genome $(\sim 98 \%)$ is non-coding and contains regulatory elements (e.g. enhancers and promoters) that influence cell/tissue-type specific processes [2]. Methodologically, whole genome sequencing (WGS) allows for genomewide discovery of somatic variation and may identify novel non-coding driver mutations. Recent studies of non-coding somatic variation identified recurrent mutations in the TERT promoter across several cancer types [3, 4] and the FOXA1 promoter in breast cancer [5]. A variety of computational tools are available for somatic analysis of protein-coding genes $[1,6]$, while fewer exist for interrogating the non-coding genome. Though studies have

(c) The Author(s). 2020 Open Access This article is licensed under a Creative Commons Attribution 4.0 International License, which permits use, sharing, adaptation, distribution and reproduction in any medium or format, as long as you give appropriate credit to the original author(s) and the source, provide a link to the Creative Commons licence, and indicate if changes were made. The images or other third party material in this article are included in the article's Creative Commons licence, unless indicated otherwise in a credit line to the material. If material is not included in the article's Creative Commons licence and your intended use is not permitted by statutory regulation or exceeds the permitted use, you will need to obtain permission directly from the copyright holder. To view a copy of this licence, visit http://creativecommons.org/licenses/by/4.0/. The Creative Commons Public Domain Dedication waiver (http://creativecommons.org/publicdomain/zero/1.0/) applies to the data made available in this article, unless otherwise stated in a credit line to the data. 
devised a variety of analytical strategies for interrogating non-coding somatic mutations, software packages implementing these routines are generally not readily available $[4,5,7]$. Existing tools capable of analyzing this space include OncodriveFML, which permutes variant impact data to assess mutation burden [8], fishHook, which employs a GammaPoisson regression framework to model somatic mutation counts along with genomic covariates [9], and MOAT, which permutes regional annotations and mutation locations to identify loci with significant mutation burdens [10].

Here, we describe MutEnricher, a flexible toolset that performs somatic mutation enrichment analysis of both protein-coding and non-coding genomic loci from WGS data. MutEnricher computes both overall mutational burden and "hotspot" enrichments in its analytical routines. MutEnricher is composed of two distinct analysis modules: 1) coding, which identifies genes harboring recurrent non-silent somatic mutations (applicable to both WES and WGS data) and 2) noncoding, which identifies enrichment of somatic variation in user-defined non-coding genomic regions. MutEnricher also implements several methods for computing background mutation rates, including a clustering procedure that groups features (i.e. genes or non-coding regions) by user-defined genomic covariates (e.g. replication timing, sequence GC content, etc.). MutEnricher is implemented in Python (compatible with both major versions 2 and 3), is parallelizable, and is highly configurable to users' specific datasets.

\section{Implementation}

Overview

MutEnricher (Fig. 1) performs somatic mutation enrichment analyses via two distinct modules accessible through the main run script (mutEnricher.py): the coding

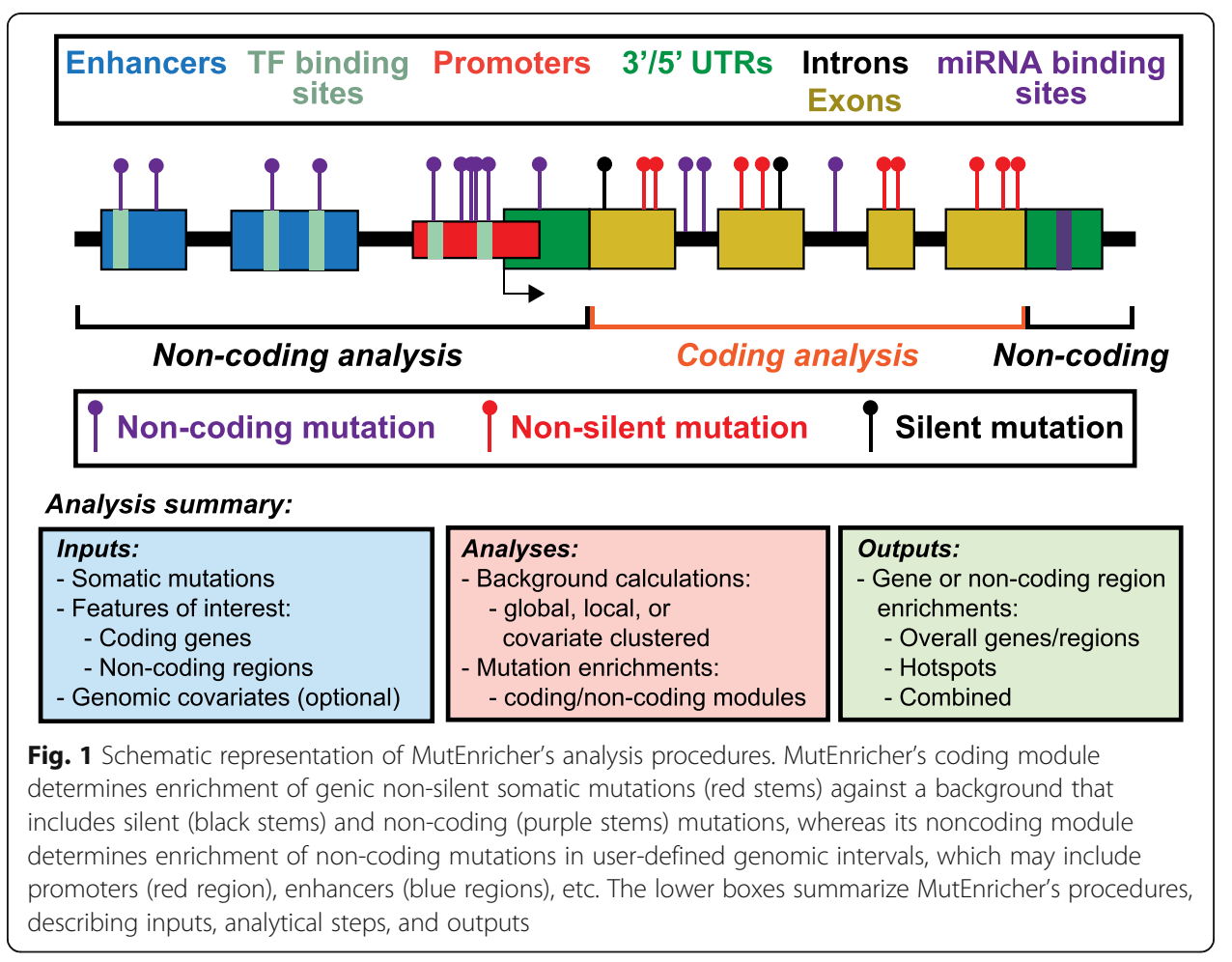


module, which assesses enrichment of non-silent somatic mutations within coding gene sequences, and the noncoding module, which determines somatic enrichment within user-defined genomic regions (e.g. promoters or enhancers). Both modules compute overall feature (i.e. gene or non-coding region) burden and "hotspot" enrichment significances using binomial statistics by default, with background mutation rates calculated according to one of several user-selected methods. While MutEnricher is designed to work with whole genome somatic mutation calls, the coding module is also capable of analyzing targeted or WES data. Both MutEnricher modules report independent burden and hotspot $p$-values along with combined significance estimates for interrogation by investigators. General run and methodological information are provided here while further details are provided in the Supplementary Information.

\section{Required inputs and file formats}

Somatic mutation data is provided to MutEnricher as a list of bgzip-compressed and tabix-indexed somatic variant call format (VCF) files. Coding gene impact annotations (e.g. via ANNOVAR [11]) are required for the coding module to distinguish nonsilent versus silent mutations, while no such annotations are required for the noncoding module. MutEnricher interrogates somatic mutation densities in user-defined features of interest; in the coding module, these are coding genes and are provided to MutEnricher with a gene transfer format (GTF) file, while features of interest in the noncoding module are genomic regions defined in an input BED format file. MutEnricher's coding module is also capable of accepting mutation annotation format (MAF) files containing mutations derived from targeted or WES data.

\section{Background mutation rate calculations}

MutEnricher implements several methods from which users can select for computing background mutation rates, which are necessary for gene and region enrichment calculations. Three main methods are available: 1) global, 2) local, and 3) covariate clustered. With the global method, gene/region backgrounds are computed as the sum of sample somatic mutation counts within all features divided by the total length; thus, with this method, all features within a sample have the same background rate. For the second method, a local background mutation rate is calculated per-gene/region for each sample. Here, local windows (1-2 Mb in length) are scanned around each feature in each sample and the background mutation rate for the samples' feature is set to the maximal observed rate from this procedure. The third method clusters features by similarity of user-supplied genomic covariates (e.g. GC content, replication timing, etc.) using affinity propagation [12] and calculates per-sample and per-feature rates from the mutation densities of cluster members. MutEnricher supplies utilities to assist users in creating covariate input files for both modules. Finally, an additional method is implemented that combines the behaviors of the local and covariate clustering methods, whereby features are again grouped by genomic covariates but a wider, local window is scanned when determining mutation densities. For all genes/regions, the final background mutation rate is calculated as the geometric mean of sample-wise rates for all samples possessing at least one foreground somatic mutation (non-silent mutation in coding analysis or any mutation in non-coding analysis) in the features of interest. 


\section{Burden and "hotspot" statistical testing}

MutEnricher implements two statistical strategies for determining somatic mutation enrichments. The first method (the default) uses the binomial distribution to determine the significance of observing $n$ samples containing somatic mutations in a given feature of length $L$ with background probability $p_{n}$ :

$$
\text { Binomial }_{\mathrm{p}-\text { value }}=\sum_{i=n}^{N}\left(\begin{array}{c}
N \\
i
\end{array}\right) p_{n}{ }^{i}\left(1-p_{n}\right)^{(N-i)}
$$

Here, $N$ is the total number of samples analyzed and $p_{n}$ is determined from the estimated nucleotide mutation rate $p$ obtained from one of the available background calculation methods and the length of the feature $L$ :

$$
p_{n}=1-(1-p)^{L}
$$

In the coding module, $n$ is the number of samples with at least one non-silent somatic mutation in a gene, while $n$ in the noncoding module is the number of samples with any observed somatic mutation in a region.

MutEnricher also allows users to compute gene/region enrichment statistics using a negative binomial testing strategy that determines the significance of observing $k$ mutations within a given feature of length $L$ and background mutation rate $p$ among $N$ samples according to:

$$
\mathrm{NB}_{\mathrm{p}-\text { value }}=\sum_{r=0}^{x-k}\left(\begin{array}{c}
k+r-1 \\
r
\end{array}\right) p^{k}(1-p)^{r}
$$

In the coding module, $k$ is the total number of non-silent somatic mutations found within a gene and $x$ is the gene's coding length multiplied by the total number of tested samples (i.e. $x=L \times N$ ). In the noncoding module, $k$ is the total number of somatic mutations found within the region and $x$ is the length of the region multiplied by the total number of samples.

In addition to computing overall gene/region burden enrichments, MutEnricher also finds significant "hotspot" enrichments by progressively grouping somatic mutations within short linear distances (e.g. 50 base pairs, a user-defined parameter) and directly testing these sub-regions for significance (similar to [4]). The noncoding module additionally implements a permutation-based weighted average proximity (WAP) scheme (described in [5]) as another test for mutation clustering. MutEnricher reports both independent burden and hotspot $p$-values along with combined significance estimates using Fisher's method. All statistical tests are subsequently corrected for multiple hypotheses using the Benjamini-Hochberg FDR procedure [13].

\section{Datasets, run characteristics, and comparisons to existing tools}

We obtained several somatic MAF files from TCGA cohorts and ran MutEnricher's coding module on these in an exome-specific mode. During these runs, we required candidate hotspots to have at least five somatic mutations from at least three patients. We compared MutEnricher results from each cancer type against results from MutSigCV [14], MutSig2CV [15], fishHook [9], and OncodriveFML [8]. We also obtained breast, liver, and medulloblastoma whole genome somatic mutation data from 
[16] to test both MutEnricher's coding and noncoding modules on WGS data. We compared MutEnricher coding results on these data to fishHook and OncodriveFML, and additionally compared noncoding module results to MOAT [10]. Further details are provided in the Supplementary Information and analysis run times on synthetic WGS data are reported in Supplementary Table 1.

\section{Results and discussion}

We ran MutEnricher's coding module on seven WES-derived mutation datasets from TCGA and compared these results to MutSigCV, MutSig2CV, fishHook, and OncodriveFML significance calls (corrected $p$-values $<0.01$ ). Overall, we observed strong overlap among genes called statistically significant by MutEnricher's burden testing strategy with those also called by MutSigCV (100\% median/ 76\% mean overlap, dataset-wise overlap significance all $<2.8 \mathrm{e}-4$ by hypergeometric test, Supplementary Table $2 \mathrm{~A}$ ). Genes not identified as significant by direct burden testing but significant when hotspots were considered include KRAS in BRCA (burden FDR $=1$, combined FDR = 4.2e-7) and BRAF in GBM (burden FDR $=1$, combined FDR $=7.8 \mathrm{e}-12$ ). We compared MutEnricher's combined burden and hotspot results to MutSig2CV significance calls, which considers three types of evidence levels, and again found substantial overlap between significant gene calls (69\% median/55\% mean overlap, Supplementary Table 2B). MutEnricher burden results were also consistent with fishHook results (81.8\% median/ 66.3\% mean overlap, Supplementary Table 2C) and, to a lesser degree, with OncodriveFML (62.5\% median/53.5\% mean overlap, Supplementary Table 2D); these latter results are likely a consequence of the distinct variant impact permutation strategy employed by OncodriveFML. Overall, results from these tools on TCGA lung datasets (LUAD and LUSC) were highly variable (e.g. 539 significant genes in LUAD by MutSigCV, 4 by fishHook); MutEnricher's consistency with all tools was higher when these cancer types were not considered (Supplementary Tables 2A-D).

We next tested MutEnricher's coding and noncoding modules on breast, liver, and medulloblastoma whole genome somatic mutation calls from Alexandrov et al. [16]. We compared coding and non-coding analysis results to fishHook and OncodriveFML and additionally tested non-coding results against MOAT's annotation-based algorithm. Significantly mutated genes called by MutEnricher and fishHook were highly consistent (87.5\% median/84.7\% mean overlap), while OncodriveFML results differed from both tools (Supplementary Tables 3A-B). Genes called by MutEnricher and fishHook included TP53, GATA3, and PIK3CA in breast, TP53 and ALB in liver, and $D D X 3 X$ and $S M O$ in medulloblastoma. For non-coding analyses, we focused on liver somatic mutations as hepatocellular carcinomas are known to possess recurrent hotspot mutations in the TERT promoter [17]. We used two separate definitions for gene promoters in these analyses: 1) 100 basepairs immediately upstream of gene transcription start sites (TSSs) and 2) 2 kilobases upstream of gene TSSs with extension into the $5^{\prime}$ untranslated region (5' UTR). With the short promoter definition, all tools, with the exception of OncodriveFML, identified the TERT promoter as highly statistically significant (Fig. 2, Supplementary Table 4). Five samples in this cohort possess a TERT proximal promoter hotspot mutation that creates an ETS binding site [17]. With the longer promoter definition, the reported significance levels for this region dropped for most tools, often beyond thresholds for significance. MutEnricher's hotspot 


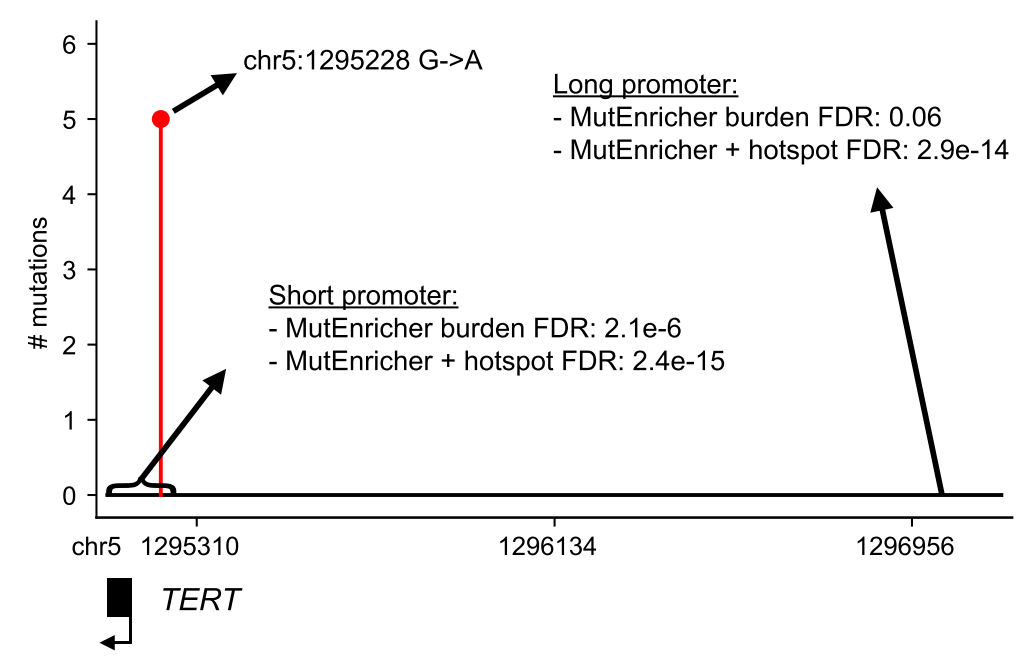

Fig. 2 TERT promoter region (hg19 chr5:1295105-1,295,262 short region; chr5:1295105-1,297,162 long region) displaying hotspot somatic mutations (chr5:1295228 G $\rightarrow$ A) identified in liver cancer whole genome datasets. MutEnricher full region (burden) and full promoter plus hotspot (+ hotspot) significance calls are also displayed

detection method, however, helped identify this longer TERT promoter region as highly significant (combined burden plus hotspot FDR $=2.95 \mathrm{e}-14$ ). Thus, MutEnricher is less sensitive to specific boundary definitions of non-coding regulatory elements because it interrogates overall regional burdens as well as hotspots.

\section{Conclusions}

Here, we presented MutEnricher, an open source, highly customizable, and well documented toolset that analyzes both coding and non-coding somatic mutation enrichment. We demonstrated general consistency between MutEnricher and other available tools that analyze the coding and/or non-coding genome on a variety of WES and WGS datasets. We also highlighted advantages of MutEnricher's combined burden and hotspot testing strategies towards driver gene and non-coding element identification. Particularly with respect to non-coding elements, this combined strategy enables MutEnricher to robustly identify mutation recurrence from variable definitions of the same regulatory elements (e.g. "short" versus "long" promoters). In addition, MutEnricher can be run quickly with a variety of parameters, allowing users to test various assumptions and alternative hypotheses. As WGS is increasingly being employed to identify novel somatic alterations, particularly towards the study of many cancers, we believe MutEnricher will be a valuable analytical tool for the research community.

\section{Availability and requirements}

Project name: MutEnricher

Project home page: https://github.com/asoltis/MutEnricher

Operating system: Linux, macOS (source code); Docker-compatible systems

Programming language: Python 2 \& 3

License: MIT license

Any restrictions to use by non-academics: None 


\section{Supplementary information}

Supplementary information accompanies this paper at https://doi.org/10.1186/s12859-020-03695-z.

Additional file 1. MutEnricher Supplementary Information. MutEnricher supplementary methods and results.

\section{Abbreviations}

WGS: Whole genome sequencing; WES: Whole exome sequencing; VCF: Variant call format; BED: Browser extensible data; GTF: Gene transfer format; MAF: Mutation annotation format; Mb: Megabase; WAP: Weighted average proximity; FDR: False discovery rate; TCGA: The Cancer Genome Atlas; TSS: Transcription start site; UTR: Untranslated region

\section{Acknowledgments}

The authors would like to acknowledge all beta testers of the MutEnricher software.

\section{Disclaimer}

The contents of this publication are the sole responsibility of the author(s) and do not necessarily reflect the views, opinions or policies of Uniformed Services University of the Health Sciences (USUHS), the Department of Defense (DoD), The Henry M. Jackson Foundation for the Advancement of Military Medicine, Inc., or the U.S. Government.

\section{Authors' contributions}

ARS and MDW conceived of the project. ARS designed, wrote, and tested the MutEnricher software and performed all analyses. CLD and HBP provided programmatic support to the project. ARS wrote the manuscript with assistance from MDW. MDW supervised the overall project. All authors have read and approved the final manuscript.

\section{Funding}

This study was supported by NHLBI Grant IAA-A-HL-007.001. This entity had no part in the design of this software nor the collection, analysis, or interpretation of data or composition of the manuscript.

\section{Availability of data and materials}

Synthetic WGS datasets, gene models, and non-coding element definitions are available as example data on the MutEnricher GitHub page (https://github.com/asoltis/mutenricher); TCGA exome MAF files are available from the Broad Institute's Firehose server (https://gdac.broadinstitute.org/); WGS cancer mutation datasets are available at ftp://ftp. sanger.ac.uk/pub/cancer/AlexandrovEtAl/somatic_mutation_data.

\section{Ethics approval and consent to participate}

Not applicable.

\section{Consent for publication}

Not applicable.

\section{Competing interests}

The authors declare that they have no competing interests.

\section{Author details}

${ }^{1}$ The American Genome Center, Collaborative Health Initiative Research Program, Uniformed Services University of the Health Sciences, Bethesda, MD, USA. ${ }^{2}$ Henry M. Jackson Foundation for the Advancement of Military Medicine, Bethesda, MD, USA. ${ }^{3}$ Department of Anatomy, Physiology, and Genetics, Uniformed Services University of the Health Sciences, Bethesda, MD, USA.

Received: 15 April 2020 Accepted: 21 July 2020

Published online: 31 July 2020

\section{References}

1. Bailey MH, Tokheim C, Porta-Pardo E, Sengupta S, Bertrand D, Weerasinghe A, Colaprico A, Wendl MC, Kim J, Reardon B, et al. Comprehensive characterization of cancer driver genes and mutations. Cell. 2018;173(2):371-385.e318.

2. Khurana E, Fu Y, Chakravarty D, Demichelis F, Rubin MA, Gerstein M. Role of non-coding sequence variants in cancer. Nat Rev Genet. 2016;17(2):93-108.

3. Horn S, Figl A, Rachakonda PS, Fischer C, Sucker A, Gast A, Kadel S, Moll I, Nagore E, Hemminki K, et al. TERT promoter mutations in familial and sporadic melanoma. Science. 2013;339(6122):959-61.

4. Weinhold N, Jacobsen A, Schultz N, Sander C, Lee W. Genome-wide analysis of noncoding regulatory mutations in cancer. Nat Genet. 2014;46(11):1160-5.

5. Rheinbay E, Parasuraman P, Grimsby J, Tiao G, Engreitz JM, Kim J, Lawrence MS, Taylor-Weiner A, Rodriguez-Cuevas S, Rosenberg M, et al. Recurrent and functional regulatory mutations in breast cancer. Nature. 2017;547(7661):55-60.

6. Tokheim CJ, Papadopoulos N, Kinzler KW, Vogelstein B, Karchin R. Evaluating the evaluation of cancer driver genes. Proc Natl Acad Sci U S A. 2016;113(50):14330-5.

7. Nik-Zainal S, Davies H, Staaf J, Ramakrishna M, Glodzik D, Zou X, Martincorena I, Alexandrov LB, Martin S, Wedge DC, et al. Landscape of somatic mutations in 560 breast cancer whole-genome sequences. Nature. 2016:534(7605):47-54

8. Mularoni L, Sabarinathan R, Deu-Pons J, Gonzalez-Perez A, Lopez-Bigas N. OncodriveFML: a general framework to identify coding and non-coding regions with cancer driver mutations. Genome Biol. 2016;17(1):128.

9. Imielinski M, Guo G, Meyerson M. Insertions and deletions target lineage-defining genes in human cancers. Cell. 2017; 168(3):460-472.e414 
10. Lochovsky L, Zhang J, Gerstein M. MOAT: efficient detection of highly mutated regions with the mutations overburdening annotations tool. Bioinformatics. 2017;34(6);1031-3.

11. Wang K, Li M, Hakonarson H. ANNOVAR: functional annotation of genetic variants from high-throughput sequencing data. Nucleic Acids Res. 2010;38(16):e164.

12. Frey BJ, Dueck D. Clustering by passing messages between data points. Science. 2007;315(5814):972-6.

13. Benjamini $Y$, Hochberg $Y$. Controlling the false discovery rate: a practical and powerful approach to multiple testing. J R Stat Soc Series B. 1995;57(1):289-300.

14. Lawrence MS, Stojanov P, Polak P, Kryukov GV, Cibulskis K, Sivachenko A, Carter SL, Stewart C, Mermel CH, Roberts SA, et al. Mutational heterogeneity in cancer and the search for new cancer-associated genes. Nature. 2013;499(7457):214-8.

15. Lawrence MS, Stojanov P, Mermel CH, Robinson JT, Garraway LA, Golub TR, Meyerson M, Gabriel SB, Lander ES, Getz G. Discovery and saturation analysis of cancer genes across 21 tumour types. Nature. 2014;505(7484):495-501.

16. Alexandrov LB, Nik-Zainal S, Wedge DC, Aparicio SA, Behjati S, Biankin AV, Bignell GR, Bolli N, Borg A, Borresen-Dale AL, et al. Signatures of mutational processes in human cancer. Nature. 2013;500(7463):415-21.

17. Nault JC, Mallet M, Pilati C, Calderaro J, Bioulac-Sage P, Laurent C, Laurent A, Cherqui D, Balabaud C, Zucman-Rossi J. High frequency of telomerase reverse-transcriptase promoter somatic mutations in hepatocellular carcinoma and preneoplastic lesions. Nat Commun. 2013;4:2218.

\section{Publisher's Note}

Springer Nature remains neutral with regard to jurisdictional claims in published maps and institutional affiliations.

\section{Ready to submit your research? Choose BMC and benefit from}

- fast, convenient online submission

- thorough peer review by experienced researchers in your field

- rapid publication on acceptance

- support for research data, including large and complex data types

- gold Open Access which fosters wider collaboration and increased citations

- maximum visibility for your research: over $100 \mathrm{M}$ website views per year

At $\mathrm{BMC}$, research is always in progress.

Learn more biomedcentral.com/submissions 